\title{
Dissolution Highlights from the 2017 AAPS Annual Meeting in San Diego
}

\author{
Nikoletta Fotaki ${ }^{1,}{ }^{*}$, Johannes Krämer ${ }^{2}$, Xujin Lu $^{3}$, Dorys Diaz ${ }^{4}$, and Connie Langer ${ }^{4}$ \\ ${ }^{1}$ Department of Pharmacy and Pharmacology, University of Bath, Bath, UK \\ ${ }^{2}$ Eurofins-PHAST, Konstanz, Germany \\ ${ }^{3}$ Drug Product Science and Technology, Bristol-Myers Squibb, New York, NY, USA \\ ${ }^{4}$ Global Chemistry, Manufacturing and Controls, Pfizer Inc., Groton, CT, USA
}

\section{INTRODUCTION}

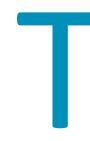
he American Association of Pharmaceutical Scientists (AAPS) held its Annual Meeting and Exposition at the Convention Center in San Diego, CA, November 12-15, 2017. Pre-conference workshops and short courses took place November 11-12, 2017. The meeting is a premier gathering of pharmaceutical scientists from around the world and works to address the needs of the attendees, including members of over 40 focus groups from nine sections, including two sections focused on dissolution testing (In vitro Release and Dissolution Testing and QbD and Product Performance).

For those with an interest in dissolution testing, there was a pre-conference short course on "Predictive Dissolution Modeling: Next-Generation Development and Release Strategy," a symposium on "Spectroscopic Imaging for In vitro Dissolution and Formulation Characterization," and a dialog and debate session on "Clinically Relevant Dissolution Specifications-Is this Really Possible?" There were face-to-face meetings of the two focus groups mentioned and the Dissolution Discussion Group. Details arising from these events are presented in this review.

\section{SHORT COURSE: "PREDICTIVE DISSOLUTION MODELING: NEXT-GENERATION DEVELOPMENT AND RELEASE STRATEGY"}

Raimar Loebenberg (University of Edmonton, Canada) started the workshop with his presentation on "Theoretical Basis of Dissolution." Dr. Loebenberg gave a comprehensive overview of the science of dissolution testing. He started with the fundamentals of dissolution based on the solubility properties of the drug substance. First, he presented the Noyes-Whitney equation and its extensions to Nernst-Brunner. Those equations are fundamental to understand the impact of stirring, the volume of dissolution medium, and other parameters, which are frequently used to adjust the dissolution behavior of solid dosage forms. He also shared with the audience the Hixon-Crowell equation, which explains the impact of drug particle geometry and hence exposed surface on the dissolution rate. The above-mentioned principles equations can be applied to the drug substance but are not, per se, applicable to the dissolution rate of dosage forms.

In pharmaceutical technology, the dissolution kinetics may vary if mechanisms based on erosion, diffusion, or osmotic pressure is prevailing. These are based on the composition of the matrix, the physicochemical properties of a film coat, or the diameter of an orifice releasing the dissolved drug substance to the surrounding aqueous liquid. To each type of mechanism, typical shapes of dissolution profiles are related. They can be described as mathematical equations. The parameters of those equations can be fitted to the experimental data. The resulting functions are needed to define input functions into the system. Hence, blood concentrations can be modeled using software packages. Dr. Loebenberg summarized his published findings with the commercial software, DDSolver (1). The proper mathematical description of the dissolution kinetics is also helpful to quantify the effect of the manufacturing process. This was elucidated for the dissolution kinetics of an immediate-release (IR) tablet manufactured either by direct compression or compression after granulation. It is common to assume that the composition of the dissolution medium has an effect on the solubility of the drug in suspension. Dr. Loebenberg showed examples where disintegration prior to dissolution was dependent on the characteristics of the dissolution medium. With proper description of the disintegration sub-process, his research is helpful when applying $\mathrm{ICH}$ Q6A, i.e., waiving dissolution testing for market release in favor of disintegration testing for certain IR oral dosage forms. There is complexity predicting the rate-limiting step of oral absorption for each gastrointestinal (GI) segment due to factors such as $\mathrm{pH}$-dependent solubility or

${ }^{*}$ Corresponding author 
absorption at different sites of the Gl tract, making it difficult to predict the physiological changes throughout the GI passage. As required by Siewert et. al., the ideal in vitro dissolution test should allow prediction of the apparent in vivo dissolution behavior of the drug product (2). Sequential dissolution testing in combined systems is used in academia to model the in vivo conditions, and their use for prediction may require further work. Again, software provides a chance for time-saving in the lab; i.e., GastroPlus is one such software that the scientists of Loebenberg's group are using for their research. Dr. Loebenberg stated, that in vitro results combined with in silico simulations using GastroPlus scientifically supported that a biowaiver for IR etoricoxib solid oral dosage forms was justified (3).

Sandra Suarez-Sharp (US Food and Drug Administration) gave a presentation entitled "The Value of Dissolution and Real Time Release Testing (RTRT) - A Regulatory Aspect." Dr. Suarez described dissolution testing beyond its use as a quality control (QC) tool when dissolution is used a priori to predict the in vivo performance of a drug product for orally administered dosage forms (mainly to predict bioavailability). This is mentioned in the CFR 314.50 among other quality parameters. However, this is precluded in many cases by the uncertainty of the over/under-predictive ability of a particular dissolution method. Meaningful dissolution results should help to describe the relationship between critical attributes/ process parameters and the clinical outcome of a certain product. With the help of dedicated pharmacokinetic (PK) studies, mathematical relationships between the in vivo and in vitro performance of product variables can be established as classical in vitro-in vivo correlations (IVIVCS). The techniques used for that purpose include deconvoluting the in vivo plasma concentration data to obtain the in vivo dissolution rate. Beside this, convolution by mathematical modeling is of increasing importance. Dr. Suarez explained the term in silico physiology-based pharmacokinetic (PBPK) modeling. In addition, she indicated that the development of clinically relevant dissolution testing (CRDT) methods is based on a design of in vitro experiments, which requires a preliminary risk evaluation. Another topic of her presentation dealt with the key elements on the approval of dissolution models for real-time release testing (RTRT). Dr. Suarez defined RTRT as moving the QC lab into the process and measuring the critical quality attributes (CQAs) where they are generated, giving the opportunity for immediate action in case of process deviation. It was indicated that RTRT provides a high level of confidence in the product. It also provides increased manufacturing flexibility and efficiency as well as a framework for continuous manufacturing. CQAs can be attained by a variety of measurements such as online or in-line measurements or fast at-line measurements. Surrogates of dissolution testing (e.g., spectroscopic methods) require multivariate models. Dr. Suarez concluded her presentation with several examples from the FDA. She summarized the documentation considerations in regulatory submissions of RTRT models for dissolution, starting with the quality of data, including the appropriateness of sample size and data pretreatment. The subsequent model building begins with model assumptions and the justification of variable selection. Tabular or graphical summaries of model inputs and outputs should be reported. Model equations should be justified, and the model robustness should be validated. The model validation requires proper statistical analysis of data and should show fit and prediction ability as a rationale for establishing acceptance criteria. The model verification requires internal and external proof of predictability.

James K. Drennen (Duquesne University, USA) presented the results of the project "Development of Real-Time Release Testing Dissolution Models for Theophylline and Carbamazepine Tablets." His project partners outside Duquesne University were from the Lilly Research Laboratories and Lancaster Laboratory. The objective of the project was to demonstrate the prediction of the dissolution profile based on an empirical or semi-empirical model (e.g., chemometric model) that uses the combination of formulation variables (FV), the critical process parameters (CPP), and real-time measurements. He defined the most important FVs being the active pharmaceutical ingredient (API) particle size (material attributes), the characteristics of the API and the disintegrant, and the lubricant concentration. For the CPPs, blending time of lubricant, compression force, and dwell time were selected. The specimens for dissolution testing were manufactured according to different designs of experiments, such as a full factorial design, leading to approximately 50 batches for each of the model drug substances, theophylline and carbamazepine. The spectroscopic technique for the blend prior to compaction and the tablets was near infrared spectroscopy (NIRS). The dissolution kinetics were described by different mathematical equations, because the amount of disintegrant altered the shape of the dissolution profiles roughly from first- to zeroorder kinetics in the case of theophylline. Dr. Drennen concluded, for theophylline, that the disintegrant level has the most significant effect on tablet dissolution behavior. He could predict dissolution profiles with an error of less

Dissolution 
than $5 \%$ across all time points using process parameters, raw material attributes, and FVs. However, tablet NIR spectra did not show strong correlations with either Weibull parameters or fraction of drug released. For the alternative model drug substance, carbamazepine, the outcome was different. All approaches successfully predicted dissolution profiles with $\mathrm{a}<5 \%$ error across all time points. Among those, the approach using data fusion provided the best performance with the results that some of the dissolution profiles were accurately predicted for samples with API particle size variability. The comparison of the findings for the two model drugs supported the general assumption, that the relationship of FVs, CCPs, NIRS, and in vitro drug release are drug substance and product related. It worked the best for carbamazepine dissolution prediction. Dr. Drennen concluded that the statistical experimental design is critical for dissolution model development. The variance of the dissolution profiles should be large enough for appropriate modeling and future prediction, but not so great as to alter the dissolution kinetics.

Kendra K. Galipeau (Merck \& Co., Inc., USA) and Brian M. Zacour (Bristol-Myers Squibb, USA) presented results of a collaboration entitled "Predictive Dissolution Modeling for Improved Formulation and Process Understanding." They presented a joint industrial view focusing on case studies. The speakers shared their companies' current approaches and their experiences with predictive dissolution modeling. The content was a clear indicator of an increasing public awareness of the approaches to predictive dissolution. It was another example of modeling being applied for RTRT, and it demonstrated successful strategies for surrogate testing. One of the surrogate methods used disintegration testing instead of dissolution testing. The other method utilized focused beam reflectance measurement (FBRM) of tablets. Their conclusion was that with robust product and dissolution modeling, a direct linkage between process parameters, raw material attributes, and dissolution can be established. This link is helpful to enhance product understanding and evaluate risk. It is an aid in product development. Furthermore, having a link between process parameters, material attributes, and dissolution supports the development of a clinically relevant dissolution specification strategy. All of the above examples support that dissolution modeling, and surrogate testing could be used to achieve RTRT for dissolution and can even be extended to models for predicting dissolution performance of a drug product during storage. Dissolution is a critical input for a PBPK model and can help inform the bioequivalent formulation design space, i.esetting of clinically relevant specifications (CRS).

Nikolay Zaborenko (Eli Lilly and Co., USA) presented "The Use of First-Principles Approach to Streamline Dissolution Method Development." His talk went into depth regarding disintegration and dissolution models, mostly based on the fundamental equations mentioned by previous speakers. He presented different models that focused on components that provide sensitivity to different API, medium, and method properties: solubility and diffusion coefficient models can be sensitive to $\mathrm{pH}$, bile salts, ionic strength, and buffer strength; boundary layer models can be sensitive to particle size, media volume, agitation rate, vessel and impeller geometry; and disintegration models can discriminate for void fraction, hardness, and tensile strength. He suggested to start with "sink" conditions, which would limit dissolution of just one dose in a given volume. His final conclusion was that at different stages in the development of a drug product, different methods can be required as well as different models with different target sensitivities.

The pre-conference workshop was concluded by Sarah Nielsen (Janssen Supply Chain, USA) with her presentation on RTRT. Her talk summarized her experiences and major highlights of the workshop, "The path from classical release testing to RTRT". Dr. Nielsen stated that RTRT is the ability to evaluate and confirm the quality of in-process and/or final product based on process data. It typically includes a valid combination of measured material attributes and process controls, as per ICH Q8(R2). Dr. Nielsen provided examples of sensor placements and controls, starting with material feeders and controlling the feed rate and moving to blending operations equipped with NIR, physical testing of tablets (e.g., weight, thickness, and hardness). and finally, spectroscopic data to confirm quality attributes, such as assay and content uniformity. The challenge is how to justify a waiver for dissolution testing. To link dissolution kinetics to spectroscopic data, models are required. There are multiple approaches to modeling that have been used for more than a decade, including, for example, the Noyes Whitney equation. Dissolution considerations at Janssen start with identifying critical material attributes and CPPs for a formulation. Applying an appropriate design of experiments, the dissolution data are generated. Based on the critical material attributes and CPPs, predictive models are established and a selective model is validated with an independent test set. 
SYMPOSIUM: "SPECTROSCOPIC IMAGING FOR IN VITRO DISSOLUTION AND FORMULATION CHARACTERIZATION"

A symposium on "Spectroscopic imaging for in vitro dissolution and formulation development" organized and moderated by Xujin Lu (Bristol-Myers Squibb, USA) and Nikoletta Fotaki (University of Bath, UK) was held on November 15, 2017. Pankaj Shah (Bristol-Myers Squibb, USA) gave a presentation on "Spectroscopic Imaging for In vitro Drug Release and Dissolution - New Analytical Tools for Formulation Development." He started with presenting the importance of dissolution behavior to formulation design, drug delivery optimization, drug product stability studies, and QC. Then, he discussed the limitations of traditional dissolution testing, which relies on UV and high-performance liquid chromatography (HPLC) for a measurement of drug content dissolved in media and does not provide adequate information on drug release mechanisms. Recent developments in spectroscopic imaging have made it possible to directly observe the changes on, or close to, the tablet surface during dissolution and generate information on chemical and physical changes of the dosage form during drug release. Dr. Shah elaborated that at Bristol-Myers Squibb, several spectroscopic imaging techniques including UV, FTIR, and Raman imaging have all been successfully applied to characterize formulations for drug release and dissolution behavior. The information obtained from the spectroscopic imaging provides a fundamental qualitative and quantitative understanding of the formulated product and it's in vitro dissolution performance, effectively guiding formulation development. He provided several examples, including characterization of structured multilayer tablets, examination of API conversion from salts to free base or free acid during tablet dissolution, and a study of drug release behaviors of solid dispersion dosage forms.

The second presentation was given by Jesper $\emptyset$ stergaard (University of Copenhagen, Denmark) on "Optimization of Solid Oral Dosage Formulations Using Surface Dissolution Imaging and Raman Spectroscopy." He stated that analytical platforms offering real-time mechanistic information of dissolution are much needed. The increasing use of spectroscopic imaging techniques is to a large extent driven by the desire to link dissolution rates to the physical and structural changes occurring within the dosage form during dissolution and to attain a better understanding of the complex processes. He detailed the technology of UV surface dissolution imaging and presented case studies that highlighted how dissolution imaging might be used as a biopharmaceutics risk assessment tool. These studies included a combination of UV surface dissolution imaging with in situ Raman spectroscopy for assessment of hydrate formation and salt disproportionation occurring during dissolution; a drug-excipient compatibility study that captured matrix swelling; and drug release and form conversion of a drug in amorphous solid dispersions, and an excipient functional characterization in drug-HPMC blends. He also demonstrated whole dosage form imaging using UV imaging and a USP-type 4 flow-through cell. He further discussed UV imaging relative to the other imaging techniques used in dissolution testing and gave a perspective on future directions.

Michael Mantle (University of Cambridge, UK) delivered the last talk on "New Insights into Drug Diffusion and Dissolution Using Magnetic Resonance Imaging." He addressed recent research concerning the use of magnetic resonance imaging (MRI) and nuclear magnetic resonance (NMR) spectroscopy to study the dissolution of solid oral dosages and biopharmaceuticals protein cakes, which touched on two aspects of formulation characterization. $\mathrm{He}$ described the MRI/NMR imaging devices for dissolution testing and the unique application examples of ${ }^{1} \mathrm{H}$ and ${ }^{19} \mathrm{~F}$ imaging data, relaxation T2 mapping, and pulsed field gradient. The above information helps with the understanding of the internal mechanisms of drug release for both model drug delivery systems, as well as commercial pharmaceutical products. These include visualization of dissolution medium ingress into the tablet, formation of swollen glassy layer, outer gel layer, and hydrogel, and differentiation of formulations with different drug loading, polymer type and composition, and diffusion and dissolution of API in polymer in a hotmelt extrusion process. He also presented novel MRI results that enhanced understanding of the reconstitution and dissolution behaviors of protein cakes produced from the lyophilization process. He showed examples for the use of fast MRI to track the reconstitution behavior of lyophilized protein and the analysis of MRI data to monitor the evolution of protein concentration during the reconstitution process. He concluded that MRI and the other spectroscopic imaging techniques have become very handy and useful tools that expand our vision and knowledge in pharmaceutical development.

\section{DIALOG AND DEBATE SESSION: "CLINICALLY RELEVANT DISSOLUTION SPECIFICATIONS- IS THIS REALLY POSSIBLE"}

This session took place November 14, 2017. Kenneth Norris, a long-time supporter of AAPS who recently retired from Pfizer Inc., was the visionary leader behind

Dissolution 
this exciting session. This session was organized and moderated by Connie Langer (Pfizer Inc, USA) and Dorys Diaz (Pfizer Inc, USA) and featured Scott Furness (FDA, USA) and Ganapathy Mohan (Merck, Sharp and Dohme Corporation, USA). The main objective was to discuss the various views on CRS, including establishing a link between the dissolution specification and in vivo performance, as well as its implementation.

There was agreement among speakers, moderators, and attendees that the crucial purpose of CRS is to provide consistent quality to the patient and guarantee that each product has the same quality and efficacy profile every time a prescription is filled. This dialog and debate session illustrated both industry and agency perspectives on CRS and stimulated much discussion. Dr. Furness outlined the importance of considering the wider applicability of clinical relevance and specifically emphasized that it is not only about clinical data but also the applicability to the patient and the link between quality and product performance. He stated that clinical relevance is more than setting dissolution specifications or developing an IVIVC. It also includes working in a multi-disciplinary setting to establish the best product quality, the foundation upon which clinical safety and efficacy assessment depend.

Dr. Mohan shared the challenges and opportunities that industry faces with implementing CRS. Some of the questions and challenges included determining which products CRS could be applied to, when to invest resources, how much data to generate, whether to use biorelevant media for dissolution testing, when to use models and simulations, and how to deal with the lack of global acceptance of CRS. He expressed that the return on investment needs to be carefully evaluated not for just cost, development time, and resources, but also from the perspective of benefitting patients globally. As such, he indicated that we must work toward the acceptance of CRS by global regulatory authorities.

The discussion among the approximately 200 attendees covered many topics. The attendees agreed that there is an opportunity to enhance the process of setting a specification by evaluating the clinical relevance of manufacturing changes and setting the specification through deeper process understanding. One view was that although in many cases it may be easy to make changes in the formulation such that differential dissolution behavior is observed, without clinical relevance, it is difficult to determine whether these changes will have any in vivo impact, leading to potentially over- or underdiscriminating dissolution methodologies. Therefore, the clinical relevance of these formulation variants needs to be established as part of the process of setting specifications. An alternative view was that developing a dissolution method that shows discrimination against meaningful manufacturing changes is not always achievable (e.g., rapidly dissolving robust formulations). Nevertheless, in the case where the method does show differences in dissolution behavior due to meaningful changes in those aspects associated with drug absorption (e.g., particle size, lubrication, hardness), this should be considered a valid approach to set a specification without in vivo data.

Notwithstanding, in the current budget-constrained environment, it is challenging to justify investment to develop complex IVIVC relationships that require additional resources during early phases for drug products that are subject to attrition. Further, in the past, there has been a mindset that specifications are only for chemists, formulators, engineers, or chemistry manufacturing and controls; however, clinicians and statisticians must be engaged, and CRS must be embraced in a multi-disciplinary fashion. It was expressed that global regulatory acceptance of a CRS will require a culture shift and may take many years to fully implement. A converging theme during the debate was that both industry and regulatory agencies are working toward the same goal of delivering patient-centric quality medicines. Common questions from the participants included: do we have necessary guidance and are we ready to bring this topic to the worldwide stage? We acknowledge that while we are far away from global harmonization, there has been some progress with the initiation of $\mathrm{ICH} M 9$, and more discussion on the global stage is needed with regards to CRS modeling tools and their applicability.

\section{ACKNOWLEDGEMENTS}

The authors disclosed no funding related to this article.

\section{CONFLICT OF INTEREST}

The authors disclosed no conflicts of interest related to this article.

\section{REFERENCES}

1. Zuo, J.; Gao, Y.; Bou-Chacra, N.; Löbenberg, R. Evaluation of the DDSolver Software Applications. BioMed Res. Int. 2014, 204925. DOI: 10.1155/2014/204925.

2. Siewert, M.; Dressman, J.; Brown, C. K.; Shah, V. P.; Aiache, J.M.; Aoyagi, N.; Bashaw, D.; Brown, C.; Brown, W.; Burgess, D.; Crison, J.; DeLuca, P.; Djerki, R.; Dressman, J.; Foster, T.; Gjellan, K.; Gray, V.; Hussain, A.; Ingallinera, T.; Klancke, J.; Kraemer, J.; Kristensen, H.; Kumi, K.; Leuner, C.; Limberg, J.; Loos, P.; Margulis, 
L.; Marroum, P.; Moeller, H.; Mueller, B.; Mueller-Zsigmondy, M.; Ofako, N.; Ouderkirk, L.; Parsi, S.; Qureshi, S.; Robinson, J.; Shah, V.; Siewert, M.; Uppoor, R.; Williams, R. FIP/AAPS guidelines for dissolution/in vitro release testing of novel/special dosage forms. AAPS PharmSciTech 2003, 4, 43-52. DOI: 10.1208/pt040107.
3. Okumu, A.; DiMaso, M.; Löbenberg, R. Computer simulations using GastroPlusTM to justify a biowaiver for etoricoxib solid oral drug products. Eur. J. Pharm. Biopharm. 2009, 72, 91-98. DOI: 10.1016/j.ejpb.2008.10.019. 